\title{
A transmembrane Type-2-like $\mathrm{Cu}^{2+}$ site in the $\mathrm{P}_{1 \mathrm{~B}-3^{-}}$-type ATPase CopB: implications for metal selectivity
}

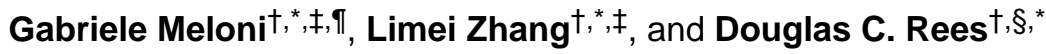 \\ tDivision of Chemistry and Chemical Engineering, California Institute of Technology, Pasadena, \\ CA 91125, USA \\ \$Howard Hughes Medical Institute, California Institute of Technology, Pasadena, CA 91125, USA
}

\section{Abstract}

Metal selectivity in $\mathrm{P}_{1 \mathrm{~B}}$-type ATPase transporters is determined by conserved amino acid residues in their transmembrane helices responsible for metal binding and transport across the cellular membrane. The $\mathrm{Cu}^{2+}$-selective $\mathrm{CopB}$ from Archaeoglobus fulgidus has been investigated to explore the coordination chemistry of the transition metal binding sites in $\mathrm{P}_{1 \mathrm{~B}-3} 3^{- \text {type }}$ ATPases. Electronic absorption, electron paramagnetic resonance and $\mathrm{X}$-ray absorption spectroscopic studies indicate the presence of a high-affinity transmembrane Type-2-like $\mathrm{Cu}^{2+}$ center in which a single cupric ion is coordinated in a distorted square pyramidal geometry by mixed nitrogen/ oxygen and sulphur ligands.

P-type ATPases are a superfamily of transmembrane primary active pumps, conserved through all the kingdoms of life, which utilize the energy generated by ATP hydrolysis to drive ion and lipid transport across the membrane ${ }^{1}$. Members of the $\mathrm{P}_{1 \mathrm{~B}}$-type class evolved as an essential transport system for the selective translocations of transition metal ions playing a fundamental role in controlling the concentrations of essential (e.g.: $\mathrm{Cu}^{+}, \mathrm{Zn}^{2+}$, $\mathrm{Co}^{2+}$ ) and toxic (e.g.: $\left.\mathrm{Ag}^{+}, \mathrm{Cd}^{2+}, \mathrm{Pb}^{2+}\right)$ metals ${ }^{2}$.

The P-type transport cycle is described by the Post-Albers scheme ${ }^{3}$. P-type pumps alternate between two functional states, E1 and E2, open to opposite sides of lipid bilayer ${ }^{3}$. Accessibility of the transported ions to transmembrane binding sites is coupled to conformational changes within the cytoplasmic domains induced by ATP binding, hydrolysis, phosphorylation of a conserved aspartic residue in the catalytic ATPase domain, and subsequent dephosphorylation ${ }^{3}$. Thus, ion(s) bind to transmembrane sites in the E1 state, are occluded within the transmembrane helices upon ATP hydrolysis and phosphorylation (E1P), and then released on the opposite side of the membrane in the E2P state $^{3}$, thereby resulting in dephosphorylation to regenerate E1 generally coupled to counterion transport. Insights in the structure and transport mechanism of P-type ATPases have been provided by crystal structures of the sarcoendoplasmatic $\mathrm{Ca}^{2+}$ pump (SERCA1a) $)^{4-5}$, the $\mathrm{Na}^{+} / \mathrm{K}^{+}$ATPase ${ }^{6-7}$, the $\mathrm{H}^{+}$pump ${ }^{8}$ and the $\mathrm{Cu}^{+}$- pump 9 .

\footnotetext{
*To whom correspondence should be addressed: gmeloni@caltech.edu, lmzhang@caltech.edu, dcrees@caltech.edu.

\$These authors contributed equally.

Il Current address: Centre for Membrane Pumps in Cells and Disease (PUMPkin), Danish National Research Foundation, Department of Molecular Biology and Genetics, Aarhus University, Gustav Wieds Vej 10C, DK-8000 Aarhus C, Denmark.

Notes

The authors declare no competing financial interest.

Supporting Information. Materials and methods, and supporting figures, tables and references. This material is available free of charge via the Internet at http://pubs.acs.org.
} 
$\mathrm{P}_{1 \mathrm{~B}}$-type ATPases typically possess 8 transmembrane helices (MA-MB-M1-6) carrying signature sequences for ion recognition (e.g.: $\mathrm{P}_{1 \mathrm{~B}-3}$-type ATPase, Suppl. Figure 1a). The transmembrane metal binding sites (TM-MBS) are essential for metal selectivity and transport across the membrane ${ }^{2}$. The requirement of conserved metal coordinating ligands for metal translocation in the transmembrane helices M4-6, including Cys, His, Glu, Asp, Ser and Met residues, has been established. Metal selectivity determined by a conserved CPX or XPC motif in M4 paralleled by specific conserved residues in helices M5-6 support

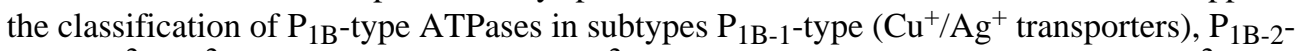
type $\left(\mathrm{Zn}^{2+} / \mathrm{Cd}^{2+}\right.$ exporters), $\mathrm{P}_{1 \mathrm{~B}-3}$-type $\left(\mathrm{Cu}^{2+}\right.$ exporters), and $\mathrm{P}_{1 \mathrm{~B}-4}$-type (probable $\mathrm{Co}^{2+}$ exporters) and $\mathrm{P}_{1 \mathrm{~B}-5}$-type (unknown selectivity) ${ }^{10}$.

As detailed structural information about the number and nature of TM-MBS in $\mathrm{P}_{1 \mathrm{~B}-3^{-}}$-type ATPases is currently lacking, we have utilized complementary biochemical and spectroscopic techniques to characterize the coordination properties of the transmembrane metal binding site in the $\mathrm{P}_{1 \mathrm{~B}-3}$-type ATPase CopB from Archaeoglobus fulgidus purified in detergent micelles. In $\mathrm{P}_{1 \mathrm{~B}-3}$ ATPases the conserved $\mathrm{CPH}$ motif in $\mathrm{M} 4$ is paralleled by the presence of conserved potential coordinating residues Asn and Tyr in M5 and Met, Ser, Thr and Asn in M6 (Suppl. Figure 1a-b) ${ }^{10}$. Sequence alignment indicates that these conserved residues strictly overlap with the residues involved in metal binding for characterized $\mathrm{Cu}^{+}$ (CopA) and $\mathrm{Zn}^{2+}(\mathrm{ZntA}) \mathrm{P}_{1 \mathrm{~B}}$-type ATPases and therefore most likely involved in transmembrane metal binding. Biochemical characterization of $\mathrm{P}_{1 \mathrm{~B}}$-type ATPases containing CPH motifs from A. fulgidus, A. aeolicus ${ }^{11}$ and T. thermophilus ${ }^{12}$ strongly indicated that these pumps are activated and involved in the transport of $\mathrm{Cu}^{2+}$ ions. Indeed, CopB from the thermophilic archea A. fulgidus was the first characterized $\mathrm{P}_{1 \mathrm{~B}-3}$-type ATPase shown to transport preferentially $\mathrm{Cu}^{2+}$ over $\mathrm{Cu}^{+}$across the lipid bilayer. ${ }^{13-14}$

In addition to the TM-MBS, regulatory cytoplasmic metal binding domains (MBDs) are present in the $\mathrm{N}$ - and/or C- cytoplasmic termini (N-MBD and C-MBD) of $\mathrm{P}_{1 \mathrm{~B}}$-type ATPases. The CopB N-MBD is not essential to confer transport activity and selectivity to the transporter ${ }^{14}$ and thus we truncated the N-MBD $(\triangle \mathrm{CopB})$ to preserve the transmembrane metal binding site(s). We performed a topology prediction with TOPCONS to identify the first transmembrane helix (Suppl. Figure 1c) $)^{15}$. The consensus from 5 different prediction algorithms identified Phe62 as the first transmembrane amino acid of MA. We thus selected Lys59 as the starting amino acid in the sequence of the $\Delta \mathrm{CopB}_{59-690}$ construct to preserve the Lys $_{59}-\mathrm{Arg}-\mathrm{Arg}_{61}$ motif which could be important for the interaction with the charged polar heads of membrane phospholipids. We fused the cloned construct to a FLAG-tag sequence for antibody-based affinity purification of $\Delta \mathrm{CopB}_{59-690}$ in 7-cyclohexyl-1-heptyl$\beta$-D-maltoside (Cymal-7) micelles. The purified $\Delta \mathrm{CopB}_{59-690}$ showed an ATPase activity maximium at around $55^{\circ} \mathrm{C}$ in agreement with thermophilic nature of A. fulgidus. A comparable ATPase activity $\left(\mathrm{V}_{\max } \sim 1.2 \mu \mathrm{mol} / \mathrm{mg} / \mathrm{h}\right)$ to the one previously observed for $\Delta \mathrm{CopB}_{55-690}$ in $\mathrm{n}$-dodecyl- $\beta$-D-maltoside (DDM) micelles and everted vesicles $\left(\mathrm{V}_{\max } \sim 2.5\right.$ $\mu \mathrm{mol} / \mathrm{mg} / \mathrm{h})^{13-14}$ was obtained. $\Delta \mathrm{CopB}_{59-690}$ also exhibited ATPase activity in the absence of $\mathrm{Cu}^{2+}$ suggesting that the energetic barrier between conformational states through the catalytic cycle might be lower in Cymal-7 micelles than in the lipid bilayer.

To identify the experimental conditions for copper binding and to determine the number of transmembrane metal binding site(s), copper titration experiments have been performed, followed by removal of unbound or weakly bound metal by size-exclusion chromatography. Metal-to-protein ratios have been subsequently determined by two independent methods, i.e. by copper quantification upon metal release using $\mathrm{Zincon}-\mathrm{Cu}^{2+}$ colorimetric measurements ${ }^{16}$ and by inductively-coupled plasma mass spectrometry (ICP-MS). In both cases the protein concentration was determined by UV absorption spectroscopy. The results from colorimetric determination $\left(\mathrm{Cu}^{2+} / \Delta \mathrm{CopB}_{59-690}=1.08 \pm 0.2 \mathrm{eq} / \mathrm{mol}\right)$ and ICP-MS 
experiments $\left(\mathrm{Cu}^{2+} / \Delta \mathrm{CopB}_{59-690}=0.70 \pm 0.1 \mathrm{eq} / \mathrm{mol}\right)$ suggested the presence of a single high-affinity transmembrane $\mathrm{Cu}^{2+}$ binding site. Similar copper binding experiments conducted under conditions of partial turnover in the presence of $\mathrm{Mg}^{2+}$-ATP or in the presence of $\mathrm{VO}_{4}{ }^{3-}$ (a P-type ATPase inhibitor that locks the pump in an E2P-like state) revealed a significant decrease in copper binding $(\sim 50 \%)$, suggesting that $\mathrm{Cu}^{2+}$ was bound to the transmembrane site responsible for $\mathrm{Cu}^{2+}$ transport (Suppl. Figure 2). Copper binding has been monitored by electronic absorption spectroscopy (Suppl. Figure 3). Upon $\mathrm{Cu}^{2+}$ binding, the absorption spectra of $\Delta \mathrm{CopB}_{59-690}-\mathrm{Cu}^{2+}$ below $350 \mathrm{~nm}$ is characterized by a complex metal-induced envelope of charge-transfer origin that overlays backbone and aromatic residues transitions indicative of ligand-to-metal charge transfer (LMCT) contributions from $\mathrm{O} / \mathrm{N}$ and/or S ligands. Metal-induced absorption intensity at $255 \mathrm{~nm}$ plotted as function of $\mathrm{Cu}$-to-protein ratio shows the presence of a breakpoint at $\sim 1 \mathrm{Cu}^{2+}$ eq. per $\Delta \mathrm{CopB}_{55-690}$, again supporting the presence of a single high-affinity transmembrane metal binding site.

Copper trafficking proteins are characterized by the presence of cuprous $\left(\mathrm{Cu}^{+}\right)$centers as a consequence of the reducing intracellular redox potential. Binding sites are dominated by digonal and trigonal coordination through methionine, cysteine and less frequently histidine residues, in which a low coordination number (usually $2-3$, more rarely 4 ) guarantee both high affinity and kinetic lability to promote $\mathrm{Cu}^{+}$transfer. In contrast, mononuclear cupric $\left(\mathrm{Cu}^{2+}\right)$ enzymatic centers in proteins where copper acts as a cofactor for redox catalysis (e.g: oxidoreductases), are classified in two groups, Type-1 or Type-2, according to their magnetic and coordination properties ${ }^{17}$. Type- 1 centers contain a single copper coordinated by His and Cys residues in a distorted tetrahedral or trigonal (bi)pyramidal structure with variable axial ligand(s), with small hyperfine splittings $\left(\mathrm{A}_{/ /}<100 \cdot 10^{-4} \mathrm{~cm}^{-1}\right)$ in the parallel region of the EPR spectrum, and strong absorption in the visible region around $600 \mathrm{~nm}$ $\left(\varepsilon \sim 5000 \mathrm{M}^{-1} \mathrm{~cm}^{-1}\right)$. Type- 2 centers are instead characterized by coordination by N/O equatorial ligands in square-planar/pyramidal geometry, and exhibit an axial EPR spectrum with hyperfine splitting similar to that observed in regular square planar or octahedral inorganic compounds $\left(\mathrm{A}_{/ /}>150 \bullet 10^{-4} \mathrm{~cm}^{-1}\right)$, together with very weak ligand-field (d-d) absorption features in the visible region $\left(\varepsilon \sim 50 \mathrm{M}^{-1} \mathrm{~cm}^{-1}\right)$. Using Peisach-Blumberg correlations it is possible to infer the nature of the ligands in Type- 2 centers ${ }^{18}$.

Due to the paramagnetic nature of transported metal by $\mathrm{CopB}\left(\mathrm{Cu}^{2+}, \mathrm{d}^{9}\right.$ metal), unusual for a protein involved in copper trafficking, we employed EPR to gain insight into the

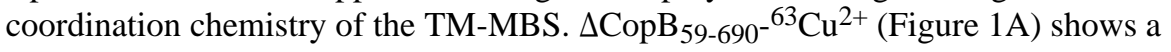
characteristic axial EPR spectrum $\left(\mathrm{g}_{\mathrm{z}}>\mathrm{g}_{\mathrm{y}} \sim \mathrm{g}_{\mathrm{x}}\right)$ with hyperfine splitting in the parallel region typical of Type- 2 centers arising from interaction with the ${ }^{63} \mathrm{Cu}$ nuclear spin (I=3/2). Spectral values of $\mathrm{g}_{\perp}=2.06, \mathrm{~g}_{/ /}=2.23$, and $\mathrm{A}_{/ /}=565 \mathrm{MHz}\left(181\right.$ Gauss or $\left.18.8 \mathrm{mK}^{18}\right)$ were obtained from the simulation of $\Delta \mathrm{CopB}_{59-690^{-}}{ }^{63} \mathrm{Cu}^{2+} \mathrm{EPR}$ spectrum. Coordination assignments using Peisach-Blumberg correlations suggest the presence of a square planar/ pyramidal site with mixed nitrogen and oxygen ligands (Figure 1B). X-band EPR analysis could not unambigously exclude the presence of one sulphur coordinating atom. Distortion from perfect planarity is indicated by spectral simulation of the perpedicular region with $\mathrm{g}_{\mathrm{x}}$ $\mathrm{g}_{\mathrm{y}}$ rhombic splitting of $\sim 0.01-0.02$. Analysis of the second derivative of the EPR spectrum reveals in the perpendicular region the presence of features separated by approx. 13-14 Gauss. These could be assigned to very weak superhyperfine features arising from the ${ }^{63} \mathrm{Cu}^{2+}$ interaction with coordinating ${ }^{14} \mathrm{~N}$ atoms (I= 1), suggesting the presence of at least $1 \mathrm{~N}$ in the coordination environment (Figure 1 inset).

In square pyramidal copper complexes, axial ligands perpendicular to the plane are more weakly bonded than equatorial ligands and do not strongly contribute to the magnetic and optical properties of the copper centers. Thus, continuous-wave X-band EPR spectroscopy is 
usually not diagnostic for the presence of coordinating axial ligands. Accordingly, complementary information on the nature of the site has been obtained from X-ray

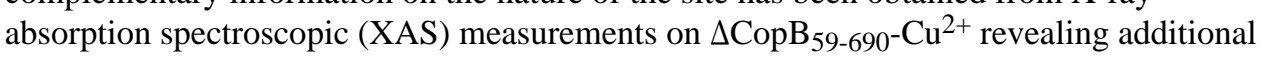
insights into $\mathrm{Cu}$ coordination and determining metal-ligand distances in the transmembrane site.

Experimental copper K-edge extended X-ray absorption fine structure (EXAFS) spectra are presented in Figure 2, together with the best fits and the corresponding EXAFS Fourier transforms. In agreement with EPR measurements, the EXAFS data could be fitted with a $\mathrm{Cu}$ complex coordinated by $4 \mathrm{~N} / \mathrm{O}$ equatorial ligands at an average distance of $1.97 \AA$ together with an additional axial S ligand at $2.68 \AA$ (Table 1, Fit 1). To investigate the possible presence of equatorial sulphur ligand(s), EXAFS data fitting was subsequently evaluated including an additional independent ligand (either $\mathrm{S}$ or N/O), in addition to 3 equatorial $\mathrm{Cu}-\mathrm{N} / \mathrm{O}$ interactions. The best EXAFS fit was obtained with three N/O ligands at $1.98 \AA$, a S ligand at $2.41 \AA$, and another S ligand at $2.68 \AA$ (Table 1 Fit 2, and Figure 2). If the equatorial $\mathrm{Cu}-\mathrm{S}$ interaction is substituted with a fourth N/O ligand, the fitting error increases (Table 1, Fit 3). The small outer shell Fourier transform peaks between $\sim 3$ and $4 \AA$ could be fit with multiple scattering arising from an imidazole ring, supporting the presence of an equatorial imidazole nitrogen coordination from the side chain of an histidine residue (Figure 2 and Table 2) ${ }^{19}$. Thus, the EXAFS results are suggestive of a square pyramidal complex with the $\mathrm{Cu}-(\mathrm{N} / \mathrm{O})$ coordination in the equatorial positions at distances of at $1.98 \AA$ typical of Type- 2 copper centers, and a possible equatorial sulphur ligand at $2.41 \AA$. The longer distance $\mathrm{Cu}-\mathrm{S}$ interaction at $2.68 \AA$ is instead consistent with distances observed for axial thioether coordination in square pyramidal copper complexes. However, considering the partial cancellation effect between the two $\mathrm{Cu}-\mathrm{S}$ components in the fitting, together with the high Debye-Waller factor obtained for the $\mathrm{Cu}-\mathrm{S}$ coordination at $2.4 \AA$, the presence of this equatorial S ligand remains uncertain. Nevertheless, in studies of model $\mathrm{Cu}^{2+}$ complexes the transformation of a 4-coordinated trigonal pyramidal complex with 1 equatorial thiolate to a square pyramidal complex resulted in elongation of the $\mathrm{Cu}-\mathrm{S}$ bond from $2.13 \AA$ (typical of type- 1 centers) to $2.45 \AA$, while changing the hyperfine interaction in EPR to the one typical of Type-2 centers, and resulting in dramatic shift the S-Cu LMCT maximum towards the UV region of the spectrum ${ }^{20}$. All these features are consistent with the data obtained on $\Delta \mathrm{CopB}_{59-690}-\mathrm{Cu}^{2+}$. The resulting distances for first shell coordination have been assessed by analysis on the filtered data, where the outer shell backscattering have been removed to exclude countributions by these components in the fitting analysis (Suppl. Table 1, and Suppl. Figure 4). The best fitting was obtained by a square pyramidal complex with mixed nitrogen and sulphur equatorial ligands as determined on the unfiltered EXAFS analysis. Furthermore, a distorted trigonal pyramidal complex with the presence of four ligands did not improve the fitting (Suppl. Table 1, Fit 4).

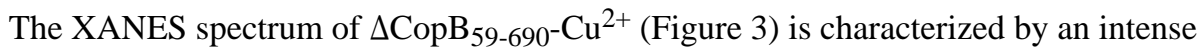
absorption feature at $8987-8988 \mathrm{eV}$, with normalized absorption of approx. 0.6 at $8988 \mathrm{eV}$, arising from a $1 \mathrm{~s} \rightarrow 4 \mathrm{p}$ transition characteristic of $\mathrm{Cu}^{2+}$ complexes. However unusual features for a $\mathrm{Cu}^{2+}$ site are observed in the spectrum, which include a low-energy feature with normalized absorption of approx 0.4 at $8984 \mathrm{eV}$, and the first inflection point determined in the first derivative spectrum at $8982 \mathrm{eV}$, which is only $\sim 1 \mathrm{eV}$ higher than the one observed in digonal and trigonal $\mathrm{Cu}^{+}$complexes. $\mathrm{Cu}^{2+}$ spin quantification by EPR performed on the XAS sample revealed the amount of $\mathrm{Cu}^{+}$to be less than $20 \%$ of the total $\mathrm{Cu}^{2+}$, thereby excluding major contribution of $\mathrm{Cu}^{+}$transitions as the origin of the peculiar XANES spectrum observed. We thus speculate that these unusual features might be in agreement with observations on "covalent" $\mathrm{Cu}^{2+}$ model complexes ${ }^{21}$ where the presence of mixed nitrogen and sulphur equatorial coordination, together with the presence of the axial sulphur ligand results in transitions at lower-energy compared to the ones of typical square 
planar $\mathrm{Cu}^{2+}$ centers with N/O ligands. Although we cannot completely rule out the possiblity of $\mathrm{Cl}^{-}$, rather than $\mathrm{S}$, as an axial ligand at the $\mathrm{Cu}^{2+}$ center by XAS technique alone, analysis of a series of Type-2-like $\mathrm{Cu}^{2+}$ model complexes in the Cambridge Structural Database suggest that $\mathrm{Cl}^{-}$would be disfavored relative to $\mathrm{H}_{2} \mathrm{O}$ as a "solvent-based" axial ligand.

Overall, the spectroscopic characterization provides evidence for a high-affinity transmembrane $\mathrm{Cu}^{2+}$ center in which a single cupric ion is coordinated in a likely distorted square pyramidal geometry by mixed nitrogen/oxygen and sulphur ligands. Equatorial histidine coordination with the possible presence of a cysteine thiolate in an equatorial position is suggested, together with the existence of axial coordination by a thioether sulphur atom. These ligands would be provided by the conserved Cys and His residues in M4 and a Met residue in M6. The two remaining equatorial ligands would be provided by the conserved residues Ser, Thr, Asn or Tyr in M5-6. Due to the peculiar spectroscopic and coordination geometry similarities between the CopB copper site and other Type- 2 centers, together with the unusual presence of mixed N/O and S ligands, we describe it as a Type-2like $\mathrm{Cu}^{2+}$ transport site. Its redox properties and possible functional implications remain to be investigated.

This study provides a detailed characterization of the transmembrane metal-binding site in a $\mathrm{P}_{1 \mathrm{~B}-3^{-}}$-type ATPase, and thereby complements the information available about sites in $\mathrm{P}_{1 \mathrm{~B}-1^{-}}$,

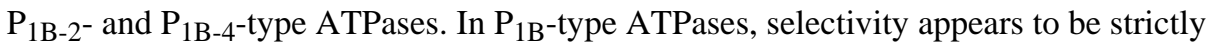
linked to the presence of a CPX or XPC motif (with X being typically, but not exclusively, a Cys, His or Ser residue) in M4 together with other family specific conserved residues in M5-6. These residues are directly involved in transmembrane ion coordination and in turn determine the substrate transport pathway. This is supported by the arrangement of these

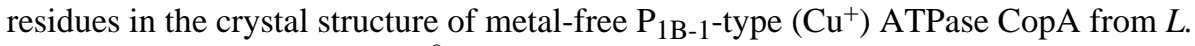
pneumophila in the E2.P $\mathrm{P}_{\mathrm{i}}$ state $^{9}$. Three residues (Met, Asp, Glu), constituting an entry site for copper, allow transport of cuprous ions to two potential transmembrane ion-binding sites which would involve the CPC motif in M6 together with Asn and Tyr on M7 and Ser and Met in $\mathrm{M} 8{ }^{9}$. In agreement, biophysical studies on the homologous CopA from A. fulgidus in the $\mathrm{E} 1$ state indicated the presence of two transmembrane trigonal planar $\mathrm{Cu}^{+}$sites with one metal coordinated by Cys380, Cys382 and Tyr682, and a second cuprous ion coordinated by Asn683, Met711, and Ser71522. Generation of the E2.P $P_{i}$ state would lead to alteration of the coordination geometry in TM-MBS resulting in translocation of the $\mathrm{Cu}^{+}$to an exit site on the periplasmic side of the transporter via an ATPase-coupled mechanism similar to SERCA1a.

An informative comparison may be made between the TM-MBS in $\mathrm{Cu}^{2+}$-selective CopB and the $\mathrm{Cu}^{+}$-selective P-type ATPases CopA from A. fulgidus. While two trigonal planar $\mathrm{Cu}^{+}$transmembrane sites occur in CopA, in CopB a single $\mathrm{Cu}^{2+}$ is coordinated in a highaffinity distorted Type-2-like transmembrane site. The different coordination chemistry nature of $\mathrm{Cu}^{2+}$ requires a square planar site with the presence of axial ligand(s) at a longer distance due to Jahn-Teller distortion. $\mathrm{Cu}^{2+}$, being an intermediate Lewis acid, shows preferable coordination by "harder" ligands compared to $\mathrm{Cu}^{+}$, with the latter showing higher preference towards donors which can be more easily polarized. Oxidation state selectivity towards $\mathrm{Cu}^{2+}$ appears thus related to the mutation of a "soft" thiolate ligand in the CPC

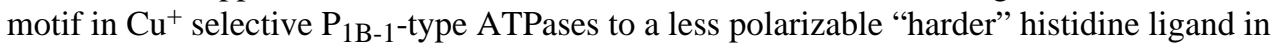
the $\mathrm{CPH}$ motif present in $\mathrm{P}_{1 \mathrm{~B}-3^{-}}$-type ATPases. $\mathrm{As}^{+} \mathrm{Cu}^{+}$favors "soft" electron pair donors like cysteine thiolate or thioether sulphurs accommodated in diagonal or trigonal coordination, the alteration of the CPC motif would therefore reduce the affinity towards $\mathrm{Cu}^{+}$ions, while the presence of the histidine would constrain the preference towards $\mathrm{Cu}^{2+}$. In addition, the conserved MXXSS motif in $\mathrm{P}_{1 \mathrm{~B}-1^{-}}$-type ATPases M6 becomes a conserved MSXST in $\mathrm{P}_{1 \mathrm{~B}-3^{-}}$ 
type ATPases which likely provides coordinating residues in a geometric arrangement which favors $\mathrm{Cu}^{2+}$ coordination. However, as a number of coordinating residues present in CopA are still present in $\mathrm{CopB}, \mathrm{Cu}^{+} / \mathrm{Ag}^{+}$will stimulate $\mathrm{P}_{1 \mathrm{~B}-3}$-type ATPase activity, although at a reduced level ${ }^{13-14}$. Considering the high sequence homology between the two proteins the comparative analysis provides insights into how metal oxidation state selectivity is partially achieved by similar $\mathrm{P}_{1 \mathrm{~B}}$-type ATPases transporters in the same microorganism. Functional and microbiological studies are required however to reveal whether and why some archaea and bacteria require the presence of primary active exporters ( $\mathrm{P}_{1 \mathrm{~B}-3}$-type ATPAses) capable of transporting cupric ions.

While a clearer understanding of the number of TM binding sites in different $\mathrm{P}_{1 \mathrm{~B}}$-type ATPase and their coordinating environment is emerging, the molecular mechanism by which conformational changes throughout the catalytic cycle control the coordination chemistry during the transport process remains elusive. Nevertheless, the revealed transmembrane coordination differences between CopA and CopB support the concept that chemical and geometric constraints imposed by conserved amino acid residues in the transmembrane helices are responsible for metal binding and coordination which result in selective transport across the cellular membranes.

\section{METHODS}

Recombinant $\triangle \mathrm{CopB}_{59-690}$ possessing an N-terminal FLAG sequence has been expressed in BL21(DE3) GOLD or Rosetta 2 E. coli strains and purified by affinity chromatography in 7 cyclohexyl-1-heptyl- $\beta$-D-maltoside (Cymal-7) micelles.

$\mathrm{Cu}^{2+}$ binding stoichiometry has been determined by colorimetric quantification using 2-[5(2-hydroxy-5-sulfophenyl)-3-phenyl-1-formazyl]benzoic acid (Zincon) and by Inductively Coupled Plasma Mass Spectrometry (ICP-MS).

Copper binding has been characterized by $\mathrm{Cu}^{2+}$ titration followed by UV-Vis absorption spectroscopy on a Cary 3 spectrophotometer (Varian Inc.)

EPR spectroscopy has been performed on purified $\Delta \mathrm{CopB}_{59-690}(60 \mu \mathrm{M})$ reconstituted with ${ }^{63} \mathrm{Cu}^{2+}$ on a X-band Bruker Elexsys 500 instrument operating at a microwave frequency of $\sim 9.3 \mathrm{GHz}$.

XAS measurements were performed on beamline 7-3 at Stanford Synchrotron Radiation Lightsource with the SPEAR 3 storage ring operated at $3.0 \mathrm{GeV}$ with the beam current of $350 \mathrm{~mA}$.

Detailed Materials and Methods are provided in the Supporting Information.

\section{Supplementary Material}

Refer to Web version on PubMed Central for supplementary material.

\section{Acknowledgments}

The work was supported by NIH (grant GM45162 to D.C.R.), by a Swiss National Science Foundation fellowship for prospective researchers (to G.M.), by a Marie Curie International Outgoing Fellowship (European Commission, grant no. 252961 to G.M.), and by a NSERC PDF award (to L.Z.). We thank Poul Nissen (University of Aarhus, Denmark) for supervising the Marie Curie Fellowship. We thank the staff at Beamline 7-3, Stanford Synchrotron Radiation Lightsource (SSRL) and staff at HXMA Beamline, Canadian Light Source (CLS). SSRL is operated for the DOE and supported by OBER and by the NIH, NIGMS (P41GM103393) and the NCRR (P41RR001209). CLS is supported by NSERC, NRC, CIHR and the University of Saskatchewan. We thank O. Lewinson and A.T. Lee for 
providing the full-length cDNA of CopB and A. Di Bilio for assistance with EPR measurements. This project benefited from discussion with Nathan Dalleska and instrumentation made available by the Caltech Environmental Analysis Center.

\section{ABBREVIATIONS}

$\begin{array}{ll}\text { ATP } & \text { adenosine-5'-triphosphate } \\ \text { Cymal-7 } & \text { 7-cyclohexyl-1-heptyl- } \beta \text {-D-maltoside } \\ \text { EPR } & \text { electron paramagnetic resonance } \\ \text { EXAFS } & \text { extended X-ray absorption fine structure } \\ \text { ICP-MS } & \text { inductively coupled plasma mass spectrometry } \\ \text { LMCT } & \text { ligand-to-metal charge transfer } \\ \text { MBD } & \text { metal binding domain } \\ \text { MES } & \text { 2-(N-morpholino) ethanesulfonic acid } \\ \text { TM-MBS } & \text { transmembrane metal binding sites } \\ \text { XANES } & \text { X-ray absorption near edge structure } \\ \text { XAS } & \text { X-ray absorption spectroscopy }\end{array}$

\section{References}

1. Bublitz M, Morth JP, Nissen P. P-type ATPases at a glance. J Cell Sci. 2011; 124:2515-2519. [PubMed: 21768325]

2. Arguello JM, Eren E, Gonzalez-Guerrero M. The structure and function of heavy metal transport $\mathrm{P}_{1 \mathrm{~B}}$-ATPases. Biometals. 2007; 20:233-248. [PubMed: 17219055]

3. Kuhlbrandt W. Biology, structure and mechanism of P-type ATPases. Nat Rev Mol Cell Biol. 2004; 5:282-295. [PubMed: 15071553]

4. Toyoshima C, Nakasako M, Nomura H, Ogawa H. Crystal structure of the calcium pump of sarcoplasmic reticulum at $2.6 \AA$ resolution. Nature. 2000; 405:647-655. [PubMed: 10864315]

5. Toyoshima $\mathrm{C}$, Nomura H. Structural changes in the calcium pump accompanying the dissociation of calcium. Nature. 2002; 418:605-611. [PubMed: 12167852]

6. Morth JP, Pedersen BP, Toustrup-Jensen MS, Sorensen TL, Petersen J, Andersen JP, Vilsen B, Nissen P. Crystal structure of the sodium-potassium pump. Nature. 2007; 450:1043-1049. [PubMed: 18075585]

7. Shinoda T, Ogawa H, Cornelius F, Toyoshima C. Crystal structure of the sodium-potassium pump at 2.4 Å resolution. Nature. 2009; 459:446-450. [PubMed: 19458722]

8. Pedersen BP, Buch-Pedersen MJ, Morth JP, Palmgren MG, Nissen P. Crystal structure of the plasma membrane proton pump. Nature. 2007; 450:1111-1114. [PubMed: 18075595]

9. Gourdon P, Liu XY, Skjorringe T, Morth JP, Moller LB, Pedersen BP, Nissen P. Crystal structure of a copper-transporting PIB-type ATPase. Nature. 2011; 475:59-64. [PubMed: 21716286]

10. Arguello JM. Identification of ion-selectivity determinants in heavy-metal transport P1B-type ATPases. J Membr Biol. 2003; 195:93-108. [PubMed: 14692449]

11. Chintalapati S, Al Kurdi R, van Scheltinga AC, Kuhlbrandt W. Membrane structure of CtrA3, a copper-transporting P-type-ATPase from Aquifex aeolicus. J Mol Biol. 2008; 378:581-595. [PubMed: 18374940]

12. Schurig-Briccio LA, Gennis RB. Characterization of the $\mathrm{P}_{\mathrm{IB}}$-Type ATPases present in Thermus thermophilus. J Bacteriol. 2012; 194:4107-4113. [PubMed: 22636781]

13. Arguello JM, Mandal AK, Mana-Capelli S. Heavy metal transport CPX-ATPases from the thermophile Archaeoglobus fulgidus. Ann N Y Acad Sci. 2003; 986:212-218. [PubMed: 12763798] 
14. Mana-Capelli S, Mandal AK, Arguello JM. Archaeoglobus fulgidus CopB is a thermophilic $\mathrm{Cu}^{2+}{ }_{-}$ ATPase: functional role of its histidine-rich-N-terminal metal binding domain. J Biol Chem. 2003; 278:40534-40541. [PubMed: 12876283]

15. Bernsel A, Viklund A, Hennerdal A, Elofsson A. TOPCONS: consensus prediction of membrane protein topology. Nucl Ac Res (Webserver issue). 2009; 37:W465-468.

16. Sabel CE, Neureuther JM, Siemann S. A spectrophotometric method for the determination of zinc, copper, and cobalt ions in metalloproteins using Zincon. Anal Biochem. 2010; 397:218-226. [PubMed: 19854146]

17. Holm RH, Kennepohl P, Solomon EI. Structural and functional aspects of metal sites in biology. Chem Rev. 1996; 96:2239-2314. [PubMed: 11848828]

18. Peisach J, Blumberg WE. Structural implications derived from the analysis of electron paramagnetic resonance spectra of natural and artificial copper proteins. Arch Biochem Biophys. 1974; 165:691-708. [PubMed: 4374138]

19. Strange RW, Blackburn NJ, Knowles PF, Hasnain SS. X-Ray absorption-spectroscopy of metal histidine coordination in metalloproteins - Exact simulation of the EXAFS of Tetrakis(Imidazole)Copper(II) Nitrate and other copper imidazole complexes by the use of a multiple-scattering treatment. J Am Chem Soc. 1987; 109:7157-7162.

20. Basumallick L, George SD, Randall DW, Hedman B, Hodgson KO, Fujisawa K, Solomon EI. Spectroscopic comparison of the five-coordinate $[\mathrm{Cu}(\mathrm{SMeIm})(\mathrm{HB}(3,5-\mathrm{iPr}(2) \mathrm{pz})(3))]$ with the four-coordinate $[\mathrm{Cu}(\mathrm{SCPh} 3)(\mathrm{HB}(3,5-\mathrm{iPr}(2) \mathrm{pz})(3))]$ : effect of coordination number increase on a blue copper type site. Inorg Chim Acta. 2002; 337:357-365.

21. Kau LS, Spira-Solomon DJ, Penner-Hahn JE, Hodgson KO, Solomon EI. X-Ray Absorption-Edge determination of the oxidation-state and coordination-number of copper - application to the Type-3 Site in Rhus Vernicifera Laccase and its reaction with oxygen. J Am Chem Soc. 1987; 109:64336442.

22. Gonzalez-Guerrero M, Eren E, Rawat S, Stemmler TL, Arguello JM. Structure of the two transmembrane $\mathrm{Cu}^{+}$transport sites of the $\mathrm{Cu}^{+}$-ATPases. J Biol Chem. 2008; 283:29753-29759. [PubMed: 18772137] 

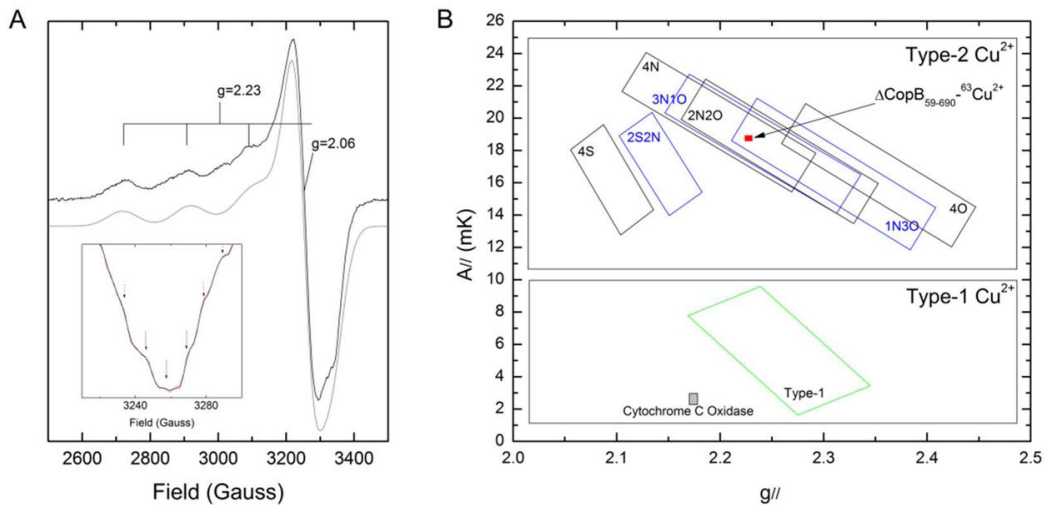

Figure 1.

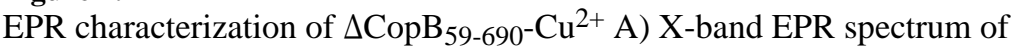
$\Delta \mathrm{CopB}_{59-690^{-}}{ }^{63} \mathrm{Cu}^{2+}(60 \mu \mathrm{M}$, black line $)$ in $20 \mathrm{mM}$ MES pH 6, $500 \mathrm{mM} \mathrm{NaCl}, 0.05 \%$ Cymal-7 (w/v) and spectral simulation (gray line). Parameters obtained from the simulation are reported in the text. In the inset the smoothed derivative of the perpendicular portion of the spectra (black line) overlaid to the raw data (red line) suggests the presence of superhyperfine interaction with ${ }^{14} \mathrm{~N}$. B) Peisach-Blumberg correlation diagram as a function of $\mathrm{Cu}^{2+}$ center type and coordinating ligands, and parameters obtained from the X-band EPR spectrum of $\Delta \mathrm{CopB}_{59-690^{-}}{ }^{63} \mathrm{Cu}^{2+} .18$ 

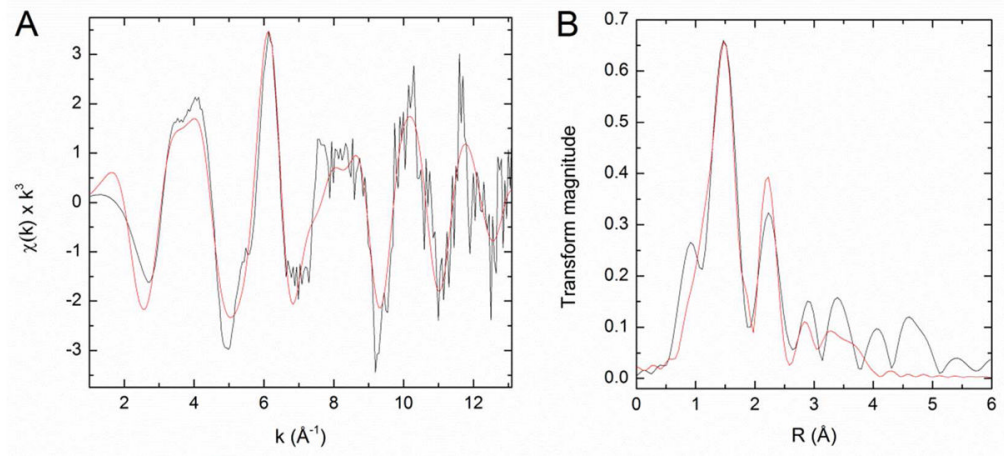

Figure 2.

X-ray absorption spectroscopy of $\Delta \mathrm{CopB}_{59-690}-\mathrm{Cu}^{2+}$ A) EXAFS experimental oscillations (black line) and corresponding calculated best fit (red line), and B) EXAFS Fourier transforms determined for $\Delta \operatorname{CopB}_{59-690}-\mathrm{Cu}^{2+}(0.35 \mathrm{mM})$ in $20 \mathrm{mM}$ MES pH 6, $500 \mathrm{mM}$ $\mathrm{NaCl}, 0.05 \%$ Cymal-7 (w/v). The parameters for the first shell coordination sphere obtained from the fittings are reported in Table 1. 


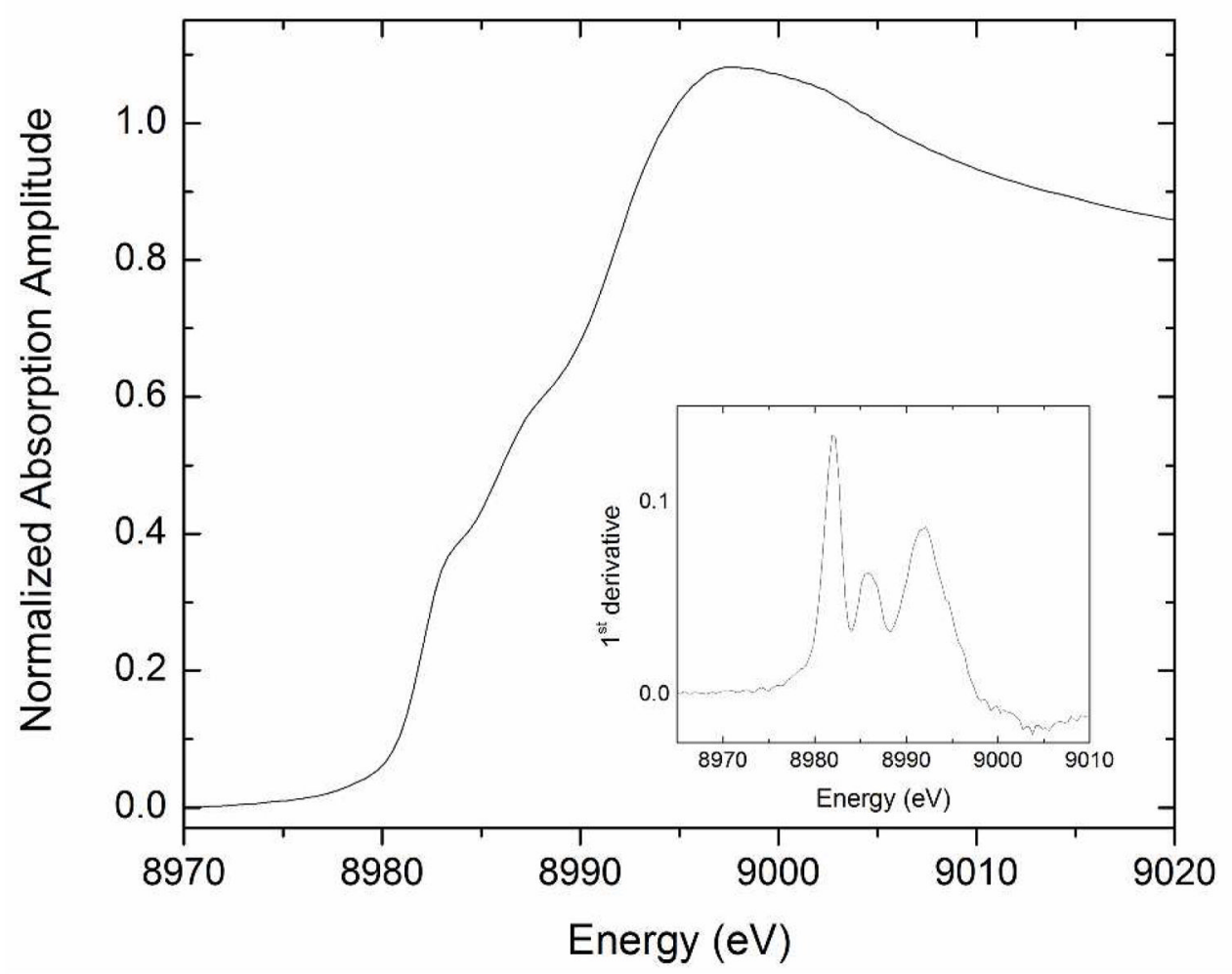

Figure 3.

XANES spectrum of $\Delta \mathrm{CopB}_{59-690}-\mathrm{Cu}^{2+}$. Normalized X-ray absorption near-edge (XANES) spectrum of $\Delta \mathrm{CopB}_{59-690}-\mathrm{Cu}^{2+}$ in $20 \mathrm{mM}$ MES pH 6, $500 \mathrm{mM} \mathrm{NaCl}, 0.05 \%$ (w/v)

Cymal-7, $20 \%(\mathrm{w} / \mathrm{v})$ glycerol. The first derivative of the XANES spectrum is presented in the inset. 


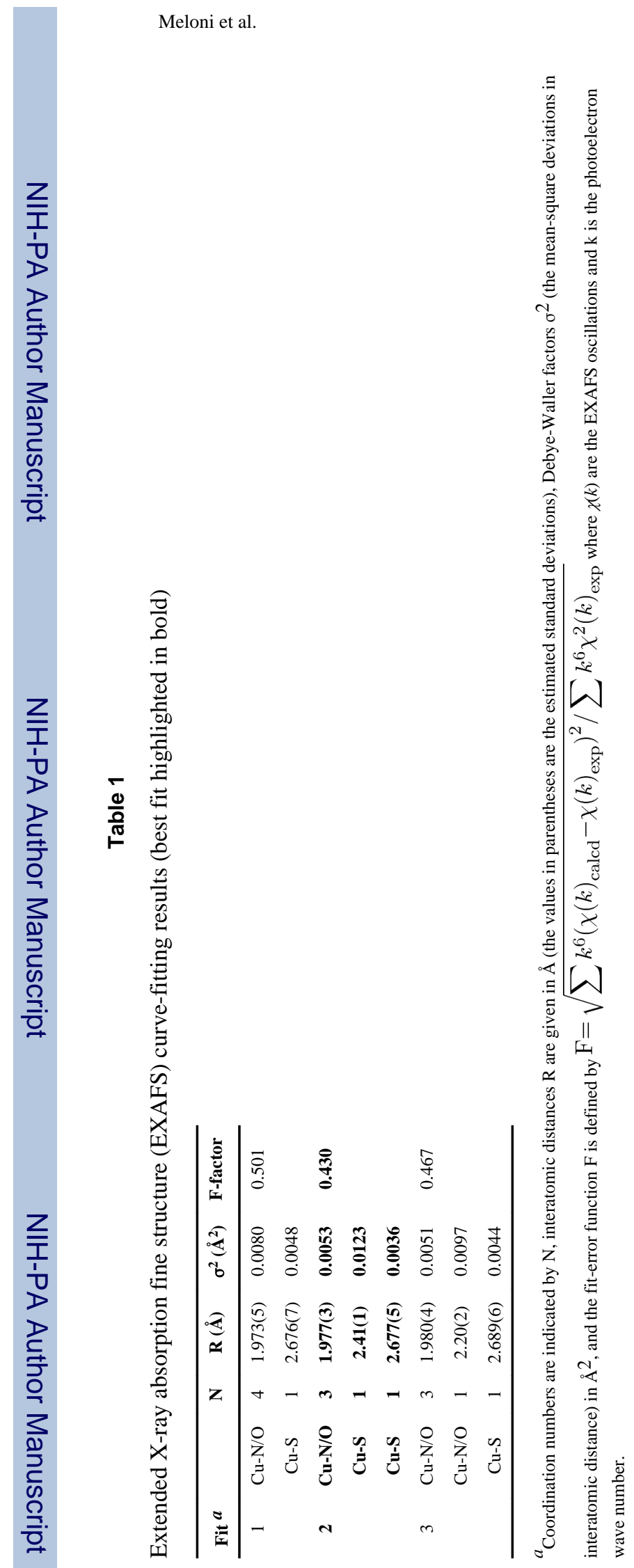

ACS Chem Biol. Author manuscript; available in PMC 2015 January 17. 